\title{
МАТЕМАТИЧЕСКОЕ МОДЕЛИРОВАНИЕ НАПРЯЖЕННО- ДЕФОРМИРОВАННОГО СОСТОЯНИЯ СВЯЗНОГО СЫПУЧЕГО МАТЕРИАЛА В КОНИЧЕСКОЙ ОБЛАСТИ
}

\author{
О. А. Фролова \\ Воронежский государственный университет, \\ ВУНЦ ВВС «Военно-воздушная академия им. проф. Н. Е. Жуковского и Ю. А. Гагарина» (г. Воронеж)
}

Поступила в редакцию 28.10.2018 г.

\begin{abstract}
Аннотация. В данной статье рассматривается модель напряженно-деформированного состояния сыпучего материала обладающего микроструктурой, который наряду со свойством сыпучести и пластичности обладает свойствами сцепления и возможностью поворота и проскальзывания отдельных частиц. Многопараметричность предложенной модели позволяет оценить особенности кинематики и напряжений в сыпучем материале, находящемся под действием силы тяжести в конусообразной емкости. Для рассматриваемой задачи получено напряженное состояние зависящее от коэффициента трения качения и угла раствора рассматриваемой области. Определена зависимость скорости перемещения от угла раствора рассматриваемой конической области.

Ключевые слова: математическое моделирование, предельное напряженное состояние, сыпучие материалы, материал с микроструктурой.

Annotation. A model of the stress-strain state of loose material is considered at present paper. The material has a microstructure and properties of flowability, plasticity, and adhesion. The multiparameter nature of the proposed model allows evaluating the kinematics and stresses in the loose material under the gravity in a cone-shaped container. The stress state depending on the rolling friction coefficient and the cone angle of the region is obtained. The dependence of the displacement velocity on the cone angle of the region is determined.

Keywords: mathematical modeling, limit stress state, loose materials, a material with microstructure.
\end{abstract}

\section{ВВЕДЕНИЕ}

Механика сыпучих материалов описывает напряженное и деформированное состояние моделей реальных материалов: песков, грунтов, гранулированных материалов, горных пород и других материалов, которые наряду со свойством пластичности и сцепления обладают свойством сыпучести, возможностью проскальзывания частиц относительно друг друга и возможностью независимого поворота отдельных частиц. Характерной особенностью таких материалов является их фрактальность, раздробленность, наличие пустот и общая пористость. Возможность движения отдельных частиц, переменность областей контакта частиц между собой порождают

() Фролова О. А., 2018 возможность течения или их жесткой консолидации в случае равновесного силового взаимодействия некоторого конечного конгломерата частиц или элементов. В работах [1-5] были рассмотрены различные подходы к исследованию сыпучих материалов.

Неоднородность сыпучих материалов, наличие зон спайки отдельных элементов в местах их контакта, ведут к различному поведению таких материалов при нагрузках, разрушающих связность элементов, и при нагрузках, разрушающих сами элементы [1]. Учет неравномерности распределения усилий на границе представительного элемента приводит к введению полного тензора напряжения, антисимметричные компоненты тензора напряжений могут быть компенсированы силами трения, которые в работе [1], при анализе условий равновесия, были опущены. Таким 
образом, осредненные усилия в гранулированных материалах приводят, в общем случае, к несимметричному тензору напряжений.

При течении сыпучего материала поведение отдельных частиц ведет к различным видам их взаимодействия: качению одного элемента по другому, взаимному проскальзыванию, нормальному контакту без перемещения. Из всего этого возникает необходимость исследования предельного состояния и течения связной гранулированной среды, при котором реализуется модель двойного скольжения - за счет относительного проскальзывания частиц и за счет их качения друг по другу $[1,6]$.

Инвариантными мерами формоизменения связных сыпучих материалов являются объемная деформация, сдвиг и вид деформирования. При этом сдвиговая и объемная деформации связаны друг с другом так, что сдвиг порождает объемную деформацию и обратно. Для связных сыпучих материалов, например, сдвиг песка приводит к переупаковке частиц, изменению порового пространства и, как следствие, к изменению объема. Свойство изменения объема материала при деформации сдвига называется дилатансией.

Существенным свойством сыпучих материалов, влияющим на течение, является независимый поворот отдельных частиц. Кинематические характеристики связных сыпучих материалов можно описать с помощью полей перемещений и независимых микровращений модельной сплошной среды.

Одним из важных моментов в описании напряженного состояния представительного элемента является предположение о наличии сил и моментов, действующих на гранях элемента, которые определяют несимметричный тензор напряжений и тензор моментных напряжений. Антисимметричность тензора напряжений и наличие моментов обусловлены кинематически независимым микровращением отдельных частиц. Микровращение частиц в различных направлениях и их перемещение друг относительно друга порождают силовое взаимодействие между частицами, определяемое трением двух типов: трением скольжения и трением качения. Течение сыпучего материала, обладающего кинематической возможностью перемещения и микровращения, может возникнуть при условии преодоления внутренних сил трения и моментов трения. Условия возникновения течения сыпучего материала могут быть сформулированы как условие пластического течения скольжения и условие микровращения.

В данной статье рассматривается модель напряженно-деформированного состояния связного сыпучего материала в конической области. Предполагается, что рассматриваемый сыпучий материал обладает многими параметрами, влияющими на его поведение. Рассматриваются алгоритмы построения поля напряжений и скоростей перемещений.

\section{ПОСТАНОВКА ЗАДАЧИ}

Положение представительного элемента сыпучей среды в пространстве будем характеризовать его перемещением и собственным поворотом. В соответствии с $[7,8]$ вводится тензор микрополярных скоростей деформации:

$$
\varepsilon_{i j}=U_{i, j}-\varepsilon_{i j k} \dot{\phi}_{k},
$$

где $U_{i}$ - скорость перемещения, $\dot{\phi}_{k}$ - угловая скорость вращения, $\varepsilon_{i j k}$ - альтернирующий тензор: $\varepsilon_{i j k}=1$ при $i, j, k$ составляющих четную перестановку из $1,2,3, \varepsilon_{i j k}=-1$ при $i$, $j, k$ составляющих нечетную перестановку из $1,2,3, \varepsilon_{i j k}=0$ в остальных случаях.

Тензор скорости деформации представляется в виде суммы симметричного тензора $\varepsilon_{(i j)}$ и антисимметричного тензора $\varepsilon_{[i j]}$ :

$$
\begin{gathered}
\varepsilon_{i j}=\varepsilon_{(i j)}+\varepsilon_{[i j]}, \\
\varepsilon_{(i j)}=\frac{1}{2}\left(U_{i, j}+U_{j, i}\right), \\
\varepsilon_{[i j]}=\frac{1}{2}\left(U_{i, j}-U_{j, i}\right)-\varepsilon_{i j k} \dot{\phi}_{k} .
\end{gathered}
$$

Антисимметричному тензору $\varepsilon_{[i j]}$ можно поставить в соответствие аксиальный вектор:

$$
\Omega_{k}=\frac{1}{2} \varepsilon_{i j k} \varepsilon_{[i j]}=\omega_{k}-\dot{\phi}_{k},
$$

где $\bar{\omega}=\frac{1}{2} \operatorname{rot} \bar{U}-$ угловая скорость вращения элемента при сдвиге, определяемая полем скоростей течения среды. 


\section{О. А. Фролова}

Сыпучая среда достигает пластического состояния, когда выполняются условия пластичности, т. е. когда напряженное и моментное состояния удовлетворяют условиям пластичности. Полагаем, что напряженное состояние пластически деформируемой микроструктурной сплошной среды соответствующее взаимному проскальзыванию представительных элементов, в пространстве полных напряжений удовлетворяет условию пластичности Мизеса - Шлейхера $[9,10]$ :

$$
\Phi_{1}=I_{2}^{\prime 2}-\left(Y+\alpha I_{1 \sigma}\right)^{2}=0,
$$

где $I_{2}^{\prime 2}=1 / 2 \cdot \sigma_{i j}^{\prime} \sigma_{i j}^{\prime}$, штрих означает девиаторную часть тензора напряжений, $Y$ - сцепление, $\alpha$ - коэффициент внутреннего трения, $I_{1 \sigma}-$ первый инвариант тензора напряжений.

Отметим, что сумма квадратов вторых инвариантов симметричной и антисимметричной частей тензора напряжений равна квадрату второго инварианта.

$$
I_{2}^{\prime 2}=I_{2(\sigma)}^{\prime 2}+I_{2[\sigma]}^{\prime 2} .
$$

Второе условие пластического течения материала, соответствующее относительному вращению частиц, сформулируем в пространстве напряжений в виде [10]:

$$
\Phi_{2}=\alpha_{1}\left(I_{2 m}^{2}-M_{0}^{2}\right)+I_{2[\sigma]}^{\prime 2}-f^{2} I_{1 \sigma}^{2}=0,
$$

где $f$ - коэффициент трения качения, $\alpha_{1}$ коэффициент, определяющий вклад моментных напряжений в поворот микрочастиц, $M_{0}$ - предельный момент пластичности при вращении частиц.

Для случая малого влияния моментных напряжений на вращение $\left(\alpha_{1} \approx 0\right)$, второе условие пластичности принимает вид:

$$
\Phi_{2}=I_{2[\sigma]}^{\prime 2}-f^{2} I_{1 \sigma}^{2}=0 .
$$

Исключая антисимметричные компоненты тензора напряжений из первого условия пластичности, получим:

$$
I_{2[\sigma]}^{\prime 2}=f^{2} I_{1[\sigma]}^{2},
$$

тогда условие пластичности примет вид:

$$
\Phi=\frac{1}{2} \sigma_{(i j)}^{\prime} \sigma_{(i j)}^{\prime}-\left(Y+\alpha \cdot I_{1 \sigma}\right)^{2}+f^{2} I_{1 \sigma}^{2}=0 .
$$

Условие пластичности в пространстве второго инварианта симметричного тензора напряжений и первого инварианта тензора напряжений представляют собой семейства: гипербол при $\alpha>f$, парабол при $\alpha=f$ или эллипсов при $\alpha<f$. При условии малости коэффициента трения качения $f \approx 0$, условие пластичности переходит в условие пластичности Мизеса - Шлейхера, которое при малом угле внутреннего трения $\alpha \approx 0$ переходит в условие пластичности Мизеса.

\section{АЛГОРИТМ ПОСТРОЕНИЯ НАПРЯЖЕННОГО СОСТОЯНИЯ В КОНИЧЕСКОЙ ОБЛАСТИ}

В сферической системе координат $r, \theta, \varphi$ рассмотрим коническую область из связного сыпучего материала с микроструктурой под действием силы тяжести (рис. 1).

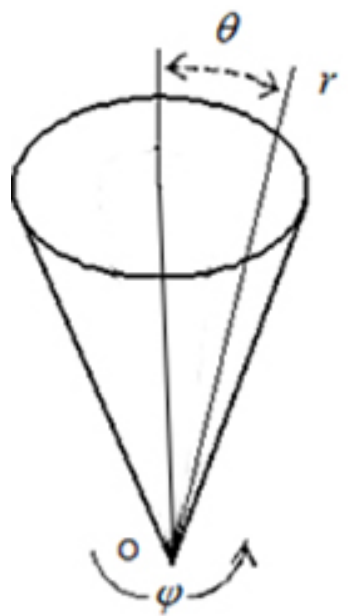

Рис. 1. Коническая область в сберической системе координат

Для напряженно-деформированного состояния, в рассматриваемой системе координат, уравнения равновесия имеют вид [11]:

$$
\begin{aligned}
& \frac{\partial \sigma_{r r}}{\partial r}+\frac{1}{r} \frac{\partial \sigma_{r \theta}}{\partial \theta}+\frac{1}{r \sin \theta} \frac{\partial \sigma_{r \varphi}}{\partial \varphi}+ \\
& +\frac{1}{r}\left[2 \sigma_{r r}-\left(\sigma_{\theta \theta}+\sigma_{\varphi \varphi}\right)+\sigma_{r \theta} \operatorname{ctg} \theta\right]=0, \\
& \frac{\partial \sigma_{r \theta}}{\partial r}+\frac{1}{r} \frac{\partial \sigma_{\theta \theta}}{\partial \theta}+\frac{1}{r \sin \theta} \frac{\partial \sigma_{\theta \varphi}}{\partial \varphi}+ \\
& +\frac{1}{r}\left[3 \sigma_{r \theta}+\left(\sigma_{\theta \theta}-\sigma_{\varphi \varphi}\right) \operatorname{ctg} \theta\right]=0, \\
& \frac{\partial \sigma_{r \varphi}}{\partial r}+\frac{1}{r} \frac{\partial \sigma_{\theta \varphi}}{\partial \theta}+\frac{1}{r \sin \theta} \frac{\partial \sigma_{\varphi \varphi}}{\partial \varphi}+ \\
& +\frac{1}{r}\left[2 \sigma_{r \varphi}+2 \sigma_{\theta \varphi} \operatorname{ctg} \theta\right]=0 .
\end{aligned}
$$


Пусть напряжения не зависят от радиуса $r$ и угла $\varphi$, а зависят только от угла раствора конуса $\theta$, т. е.

$$
\begin{gathered}
\sigma_{r r}=\sigma_{r r}(\theta), \sigma_{\theta \theta}=\sigma_{\theta \theta}(\theta), \sigma_{\varphi \varphi}=\sigma_{\varphi \varphi}(\theta), \\
\sigma_{r \theta}=\sigma_{r \varphi}=\sigma_{\theta \varphi}=0 .
\end{gathered}
$$

Тогда уравнения равновесия (2) примут вид:

$$
\begin{gathered}
\frac{1}{r}\left(2 \sigma_{r r}-\sigma_{\theta \theta}-\sigma_{\varphi \varphi}\right)=0, \\
\frac{1}{r} \frac{\partial \sigma_{\theta \theta}}{\partial \theta}+\frac{1}{r}\left(\sigma_{\theta \theta}-\sigma_{\varphi \varphi}\right) \operatorname{ctg} \theta=0
\end{gathered}
$$

или

$$
\begin{gathered}
2 \sigma_{r r}-\sigma_{\theta \theta}-\sigma_{\varphi \varphi}=0, \\
\frac{\partial \sigma_{\theta \theta}}{\partial \theta}+\left(\sigma_{\theta \theta}-\sigma_{\varphi \varphi}\right) \operatorname{ctg} \theta=0 .
\end{gathered}
$$

В сферической системе координат условие пластичности (1), с учетом сделанных предположений, примет вид:

$$
\begin{gathered}
\Phi=\sigma_{r r}^{2}+\sigma_{\theta \theta}^{2}+\sigma_{\varphi \varphi}^{2}-\beta I_{1 \sigma}^{2}-2 \alpha Y I_{1 \sigma}-Y^{2}=0, \\
\beta=\frac{1}{3}+\alpha^{2}-f^{2} .
\end{gathered}
$$

Предположим, что компоненты скорости деформации и скорости перемещений удовлетворяют следующим предположениям [11]:

$$
\begin{aligned}
& U_{r}=U_{\varphi}=0, \quad U_{\theta}=r U, \\
& \varepsilon_{r r}=\varepsilon_{r \theta}=\varepsilon_{r \varphi}=\varepsilon_{\theta \varphi}=0, \\
& \varepsilon_{\theta \theta}=\frac{\partial U}{\partial \theta}, \quad \varepsilon_{\varphi \varphi}=U \operatorname{ctg} \theta .
\end{aligned}
$$

Компоненты напряжений связаны с компонентами скорости деформации ассоциированным законом пластического течения:

$$
\varepsilon_{i j}=\lambda \frac{\partial \Phi}{\partial \sigma_{i j}},
$$

где $\lambda$ - неопределенный множитель Лагранжа.

Из ассоциированного закона пластического течения с учетом условия пластичности (4) получим:

$$
\begin{gathered}
2 \lambda\left(\sigma_{r r}-\beta \mathrm{I}_{1 \sigma}-\alpha \mathrm{Y}\right)=0 \\
2 \lambda\left(\sigma_{\theta \theta}-\beta \mathrm{I}_{1 \sigma}-\alpha \mathrm{Y}\right)=\frac{\partial U}{\partial \theta}, \\
2 \lambda\left(\sigma_{\varphi \varphi}-\beta \mathrm{I}_{1 \sigma}-\alpha \mathrm{Y}\right)=U \operatorname{ctg} \theta
\end{gathered}
$$

Из первого уравнение (5), при $\lambda \neq 0$, получим выражение связывающее компоненты напряжения:

$$
\sigma_{r r}=\frac{\beta}{1-\beta}\left(\sigma_{\theta \theta}+\sigma_{\varphi \varphi}\right)+\frac{\alpha Y}{1-\beta} .
$$

Из первого уравнения (3) получим:

$$
\sigma_{r r}=\frac{1}{2}\left(\sigma_{\theta \theta}+\sigma_{\varphi \varphi}\right) .
$$

Последние два выражения позволяют определить выражение для $\sigma_{r r}$ :

$$
\sigma_{r r}=\frac{\alpha Y}{1-3 \beta}=\frac{\alpha Y}{3\left(f^{2}-\alpha^{2}\right)} .
$$

После исключения $\sigma_{r r}$ из выражения (6) получим связь между двумя компонентами напряжения:

$$
\sigma_{\varphi \varphi}=\frac{2 \alpha Y}{3\left(f^{2}-\alpha^{2}\right)}-\sigma_{\theta \theta}
$$

Из второго уравнения (3) с учетом последнего выражения получим:

$$
\frac{\partial \sigma_{\theta \theta}}{\partial \theta}+2 \sigma_{\theta \theta} \operatorname{ctg} \theta=\frac{2 \alpha Y}{3\left(f^{2}-\alpha^{2}\right)} \operatorname{ctg} \theta .
$$

Последнее уравнение, в силу зависимости от одной переменной $\theta$, является линейным неоднородным дифференциальным уравнением и может быть проинтегрировано. В результате получим выражение для компоненты напряжения $\sigma_{\theta \theta}$ :

$$
\sigma_{\theta \theta}=\frac{\frac{\alpha Y}{3\left(f^{2}-\alpha^{2}\right)} \cos 2 \theta-2 C}{\cos 2 \theta-1},
$$

где $C$ - константа интегрирования.

С учетом выражения (9) из (8) получим выражение для компоненты напряжения $\sigma_{\varphi \varphi}$ :

$$
\sigma_{\varphi \varphi}=\frac{\frac{\alpha Y}{3\left(f^{2}-\alpha^{2}\right)}(\cos 2 \theta-2)-2 C}{\cos 2 \theta-1} .
$$

Для определения константы интегрирования $C$ зададим граничное условие:

$$
\sigma_{\theta \theta}\left(\theta_{0}\right)=-p,
$$

где $p$ - давление на внешней границе.

Из выражения (9) с учетом граничного условия получим:

$$
C=\frac{\alpha Y \cos 2 \theta_{0}}{6\left(f^{2}-\alpha^{2}\right)}-p \sin ^{2} \theta_{0} .
$$

C учетом выражения для константы $C$ выражения для компонент напряжения (9) и (10) примут вид: 


$$
\begin{gathered}
\sigma_{\theta \theta}=\frac{\alpha Y\left(\cos 2 \theta_{0}-\cos 2 \theta\right)}{6\left(f^{2}-\alpha^{2}\right) \sin ^{2} \theta}-\frac{p \sin ^{2} \theta_{0}}{\sin ^{2} \theta}, \\
\sigma_{\varphi \varphi}=\frac{\alpha Y\left(2+\cos 2 \theta_{0}-\cos 2 \theta\right)}{6\left(f^{2}-\alpha^{2}\right) \sin ^{2} \theta}-\frac{p \sin ^{2} \theta_{0}}{\sin ^{2} \theta} .
\end{gathered}
$$

Таким образом, выражения (7), (11) и (12) определяют поле напряжений для рассматриваемой задачи. На рис. 2 представлены графики для компонент напряжений при угле раствора конуса $\theta_{0}=\frac{\pi}{6}$.

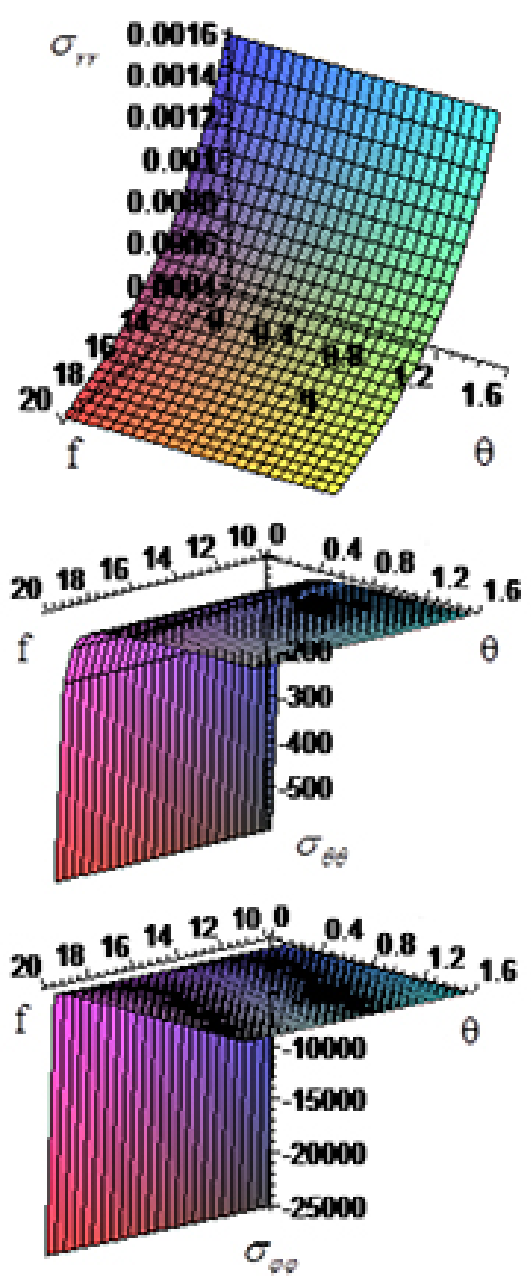

Рис. 2. Графики зависимости компонент тензора напряжений от трения качения и угла $\theta$

Из графиков рисунка 2 следует, что $\sigma_{r r}$ не зависит от угла $\theta$, а при увеличении коэффициента трения - убывает. Компонента $\sigma_{\theta \theta}$ при увеличении угла $\theta$ увеличивается, а от $f$ зависит мало. Компонента $\sigma_{\varphi \varphi}$ при увеличении угла $\theta$ резко увеличивается вначале, а потом происходит затухание увеличения, от $f$ зависит мало.

\section{АЛГОРИТМ ПОСТРОЕНИЯ ПОЛЯ СКОРОСТЕЙ ПЕРЕМЕЩЕНИЙ В КОНИЧЕСКОЙ ОБЛАСТИ}

Для определения поля скоростей перемещений рассмотрим кинематику деформирования связного сыпучего материала с микроструктурой.

Из третьего уравнения (5) выразим неопределенный множитель Лагранжа $\lambda$ :

$$
2 \lambda=\frac{U \operatorname{ctg} \theta}{\sigma_{\varphi \varphi}-\beta I_{1 \sigma}-\alpha Y} .
$$

Подставляя выражение для $\lambda$ во второе уравнение (5) получим:

$$
\frac{\partial U}{\partial \theta}=U \operatorname{ctg} \theta \frac{\sigma_{\theta \theta}-\beta \mathrm{I}_{1 \sigma}-\alpha \mathrm{Y}}{\sigma_{\varphi \varphi}-\beta \mathrm{I}_{1 \sigma}-\alpha \mathrm{Y}} .
$$

Так как выражения для компонент напряжений известны, то последнее уравнение примет вид:

$$
\frac{\partial U}{\partial \theta}=U\left(1+\frac{1}{\frac{1}{2} \cos 2 \theta-\frac{3 C\left(f^{2}-\alpha^{2}\right)}{\alpha Y}-1}\right) \operatorname{ctg} \theta .
$$

Так как в последнем уравнении функция зависит только от $\theta$, то это уравнение является обыкновенным дифференциальным уравнением и может быть проинтегрировано, в результате получим:

$$
\begin{aligned}
U=C_{1}( & -\sin \theta)^{\frac{\alpha Y-6 C\left(f^{2}-\alpha^{2}\right)}{-\alpha Y-6 C\left(f^{2}-\alpha^{2}\right)}}[\alpha Y(\cos 2 \theta-2)- \\
& \left.-6 C\left(f^{2}-\alpha^{2}\right)\right] \frac{\alpha Y}{\alpha Y+6 C\left(f^{2}-\alpha^{2}\right)},
\end{aligned}
$$

где $C_{1}$ - константа интегрирования, определяемая из граничных условий.

Последнее выражение с учетом сделанных предположений позволяет определить поле скоростей перемещений:

$$
\begin{gathered}
U_{\theta}=r C_{1}(-\sin \theta)^{\left(\alpha Y-6 C\left(f^{2}-\alpha^{2}\right)\right) /\left(-\alpha Y-6 C\left(f^{2}-\alpha^{2}\right)\right)} \times \\
\times\left[\alpha Y(\cos 2 \theta-2)-6 C\left(f^{2}-\alpha^{2}\right)\right] \frac{\alpha Y}{\alpha Y+6 C\left(f^{2}-\alpha^{2}\right)} .
\end{gathered}
$$

Как следует из рисунка 3 с увеличением угла $\theta$ перемещение $U_{\theta}$ вначале увеличивается, а достигнув определенного значения практически не меняется. 
Математическое моделирование напряженно-деформированного...

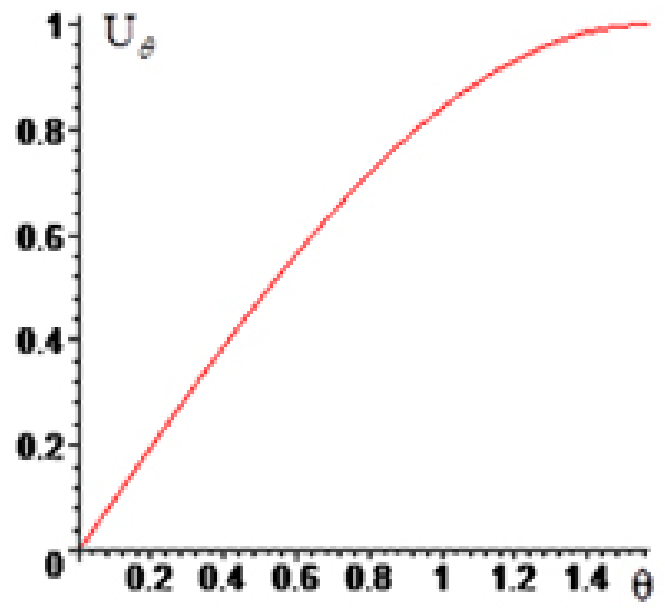

Рис. 3. Зависимость компоненты перемещения $U_{\theta}$ от угла $\theta$

\section{ЗАКЛЮЧЕНИЕ}

В предложенной работе разработан приближенно-аналитический аппарат расчета напряженно-деформированного состояния в многопараметрическом сыпучем материале, находящемся под действием силы тяготения в конусообразной области.

Для рассматриваемой области определено поле напряжений и поле скоростей перемещений. Найденные выражения в дальнейшем могут быть использованы при решении задач для конкретных материалов, за счет изменения параметров материла таких как: коэффициенты трения качения и внутреннего трения, сцепления, а также за счет изменения геометрии рассматриваемой области (угла раствора конуса).

\section{СПИСОК ЛИТЕРАТУРЫ}

1. Drescher, A. Photoelastic verification of a mechanical model for the flow of a granular material / A. Drescher, G. de Josselin de Jong // Journal of the Mechanics and Physics of Solids. 1972. - № 20. - P. 337-351.

Фролова Оксана Александровна - преподаватель, ВУНЦ ВВС «ВВА им. проф. Н. Е. Жуковского и Ю. А. Гагарина» (г. Воронеж), Воронежский государственный университет.

Тел.: +7-910-244-68-12

E-mail: OksanaFrola@yandex.ru
2. Вервейко, Н. Д. Микрополярная теория течения гранулированной среды / Н. Д. Вервейко ; Воронеж. гос. ун-т. - Воронеж, 1977. Деп. в ВИНИТИ 4.04.1978, № 1169.

3. Cambou, B. State internal variables at different scales for the modeling of the behavior of granular materials / B. Cambou, H. Magoariec, E. Vincens // Continuum Mechanics and Thermodynamics. - 2015. - № 1-2. - P. 223-241.

4. Alain, $R$. Depth and minimal slope for surface flows of cohesive granular materials on inclined channels / R. Alain, O. Louisnard // Journal of Fluid Mechanics. - 2013. - V. 727. P. 191-235.

5. Fan, Y. Kinematics of monodisperse and bidisperse granular flows in quasi-two-dimensional bounded heaps / Y. Fan, P. B. Umbanhowar, J. M. Ottino, R. M. Lueptow // Proceedings of the Royal Society of London. Series A. - 1998. 34-35. -V. 35. - P. 4597-4617.

6. Определяющие законы механики грунтов: Сб. Механика. Новое в зарубежной науке. / под. ред. В. Н. Николаевского. - Москва : Мир, 1975. - № 2. - 231 с.

7. Эринген, А. К. Теория микрополярной упругости / А. К. Эринген // Разрушение. Математические основы теории разрушения Москва : Мир. - 1975. - Т.2. - С. 646-751.

8. Ehlers, W. A Single Surface Yield Function for Geomaterials / W. Ehlers // Arch. Appl. Mech. - 1995. - P. 63-76.

9. Соколовский, В. В. Статика сыпучей среды / В. В. Соколовский. - Москва : Наука, 1990. - 272 C.

10. Валюхов, С. Г. Микрополярная модель связных сыпучих материалов / С. Г. Валюхов, Н. Д. Вервейко, О. А. Смотрова. - Воронеж : Воронеж. ун.-т, 1999.- 87 с.

11. Соколовский, В. В. Теория пластичности / В. В. Соколовский. - Москва : Высшая школа, 1969. - 608 с.

Frolova Oksana Aleksandrovna - teacher, MESC AF «N. E. Zhukovsky and Y. A. Gagarin Air Force Academy» (Voronezh), Voronezh State University.

Tel: +7-910-244-68-12

E-mail: OksanaFrola@yandex.ru 